\title{
Multiparameter sensor based on a multi- interferometric serial configuration for temperature and strain measurements
}

\author{
R. A. Perez-Herrera, M. Bravo, D. Leandro, S. Novais, J. Pradas, and M. Lopez-Amo Senior Member, \\ IEEE
}

\begin{abstract}
In this work, a multi-parameter point sensor based on the combination of Fabry-Perot (FP) and the anti-resonant (AR) reflecting guidance in cascade configuration is proposed and experimentally demonstrated. This structure, based on FP interference and $A R$ reflecting guidance, was fabricated with two different air micro-cavities. The attained experimental results showed different strain and temperature sensitivities for the antiresonance contribution. However, when analyzing the FP interference, only strain sensitivity was observed, demonstrating that this air micro-cavity was also insensitive to temperature variations.
\end{abstract}

Index Terms - Automatic optical inspection, Fabry-Perot interferometers, Multisensor systems, Optical fiber sensors, Optical interferometry.

\section{INTRODUCTION}

$\mathrm{T}$ $\mathrm{HE}$ arrival of the fourth industrial revolution has led to the emergence of new monitoring and self-diagnosis challenges for both devices' maintenance and industrial installations [1], [2]. According to this revolution, the need to evaluate many parameters in real time become of great importance for industrial processes. It becomes necessary to develop new sensor than can be integrated as a part of work environments, tool or installations. For that reasons, sensors must present characteristics such as a reduced size, have immunity against electromagnetic disturbances commonly presented in work environments, or have the possibility of carrying out real time measurements [3]. Once single-parameter measurements for attaining reliable data from an optical system have been widely reported, it has become a great challenge the development of sensors, which present the ability to discriminate and evaluate more than one parameter independently. Environmental perturbations in real-world applications and their resulting crosstalk have made this

This work was supported by the Spanish AEI projects TEC2016-76021-C2 and PID2019-107270RB-C22, FEDER Funds, the European Union's Horizon 2020 research and innovation programme under the Marie Skłodowska-Curie grant agreement No 838143, Projects for young researches UPNA 2019, "Caja Navarra" and "la Caixa" foundations under agreement LCF/PR/PR13/51080004 and the grant BGP18/00180 of the "Beatriz Galindo" programme from the Spanish Ministry of Science, Innovation and Universities.

R. A. Perez-Herrera, M. Bravo, D. Leandro, and M. Lopez-Amo are with the Electrical Electronic and Communication Engineering Department, and with the Institute of Smart Cities (ISC), Public University of Navarra, 31006 concept to become of significant importance in the past decades [4]. Multiparameter sensors based on multiple Fabry-Perot interferometers (FPI) with different cavity lengths have been previously presented [5]-[9] in which the information of the desired parameter was codified on each one of the individual cavity lengths. However, here is presented a multiinterferometric structure based on Fabry-Perot (FP) interference and anti-resonant (AR) reflecting guidance and, just by tracking the phase of the FFT of some specific spatial frequencies, the changes of the sensors can be easily monitored in real time $(1 \mathrm{~Hz})$ [10]. These phase variations reflect the wavelength shifts induced by the strain or temperature in each spectral contribution separately. This real-time FFT analysis allows isolating each characteristic frequency of the multiplexed interferometers [12], achieving a higher resolution [13] which means a remarkable robustness against unwanted parameters such as noise or dependence on polarization changes that occurs in most optical fibers.

On the other hand, some reported fiber optic interferometric sensors are designed to be relatively large in size, as well as for single-parameter measurements [14]. In this work, a simple, small, and cost-effective dual-parameter point sensor for strain and temperature measurements using FP interference and AR reflecting guidance is experimentally presented.

\section{EXPERIMENTAL SETUP}

The proposed sensor, schematically showed in Fig. 1, consists of a couple of SMF-HCF-SMF structures. A section of hollow core fiber (HCF), a capillary optical fiber with $20.5 \mu \mathrm{m}$ and $125.5 \mu \mathrm{m}$ of internal and external diameters respectively, was spliced between two sections of standard single mode fiber (SMF 28e) for each one of the two fabricated air-microcavities. The manufacturing process of these air-microcavities was

Pamplona, Spain. (e-mail: rosa.perez@unavarra.es, mikel.bravo@unavarra.es, daniel.leandro@unavarra.es,mla@unavarra.es).

J. Pradas is with the Electrical Electronic and Communication Engineering Department, Public University of Navarra, 31006 Pamplona, Spain. (e-mail: jpradas18@gmail.com)

S. Novais is with the INESC TEC-Institute for Systems and Computer Eng, Techn and Science, Rua do Campo Alegre 687, 4150-179, Porto, Portugal. (email: susana.novais@inesctec.pt) 
similar to the method described in [15]. The fusion parameters for the splicer were optimized in order to avoid collapsing or deforming the silica tube region and the discharges were mostly applied in the SMF region. Then, the silica tube was cleaved with the chosen length, under a microscope.

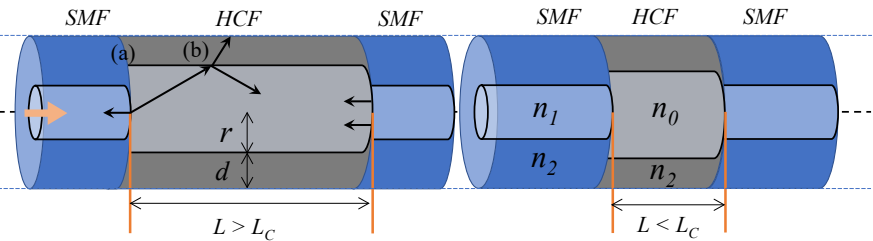

Fig. 1. Schematic diagrams of the proposed multiparameter sensor based on a cascade SMF-HCF-SMF structure.

Fig. 2 shows an image of the splice procedure for one of the SMF-HCF-SMF structures. This process was repeated by using two different HCF lengths between the SMF sections and, after that, these two air-microcavities were spliced as depicted in Fig.1.

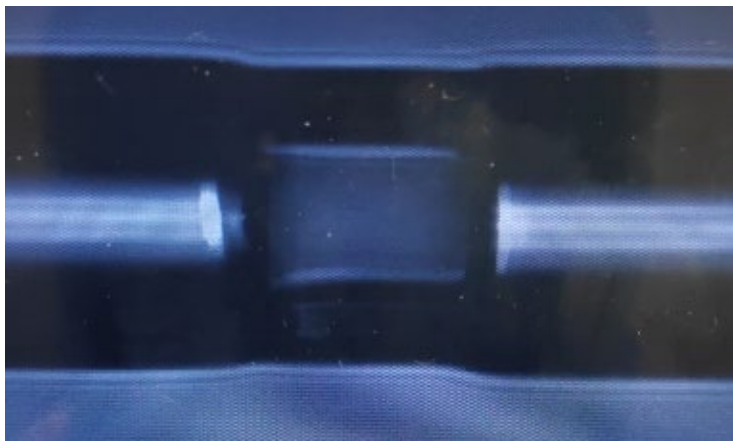

Fig. 2. Image of the splice procedure for one of the SMF-HCF-SMF structures.

Due to Fresnel reflection, a Fabry-Perot interference is generated when the inserted light is partially reflected at point (a) (see Fig. 1), that is, at the two interfaces between the SMF and the HCF. Then, the light propagates with a divergence angle and, if the length of the HCF is long enough, it reaches the cladding of the HCF (point (b), Fig. 1). At this stage, the structure can be seen as a FP interferometer along the radial direction, due to the fact that interferences between air and silica cladding behave as two reflecting surfaces [4]. After this first FP interferometer, the light waves at wavelengths than do not fulfil the resonance condition will be guided into the second and longer interferometric structure. There, the interference achieved is the corresponding to a classic SMF-MM-SMF structure [16]. In our case, an AR reflecting guidance occurs in the hollow core fiber, acting as the multimode (MM) section.

This transition between FP interference and AR reflecting guidance has been deeply analyzed in sandwiched HCF structures [17], [18], showing that when the HCF length overdoes a critical length, both interferences (FP and AR) can be observed in its reflected spectrum. As it is explained in detail in [4], the critical length for the transition between FP and AR mechanisms can be attained by using (1) [17]:

$$
L_{c}=\sqrt{n_{0}^{2}+n_{2}^{2}-n_{1}^{2}}\left(\frac{r}{\sqrt{n_{1}^{2}-n_{2}^{2}}}+\frac{2 d}{\sqrt{n_{1}^{2}-n_{0}^{2}}}\right)
$$

As Fig. 1 illustrates, $r$ is the radius of the HCF, and $d$ is the wall thickness of the cladding. On the other hand, $n_{0}, n_{l}$ and $n_{2}$ are the refractive index of air, fiber core and silica cladding, which correspond to $\sim 1,1.45$, and 1.44 in that order. Considering the dimensions of the employed HCF, $20.5 \mu \mathrm{m}$ and $125.5 \mu \mathrm{m}$ of internal and external diameters respectively, and according to (1), the critical length was calculated to be $175.9 \mu \mathrm{m}$. Taking this value into consideration, two different air micro-cavities were fabricated. The first one had a HCF length larger than the critical length, and the second one lower than the critical value that are $345 \mu \mathrm{m}$ and $165 \mu \mathrm{m}$ respectively.

After splicing these two SMF-HCF-SMF structures, a characterization of their reflection spectrum was carried out by means of a commercial FBG sensors interrogator (Micron Optics ${ }^{\circledR}$ SM 125). This multiparameter sensor was placed on a motorized translation stage (MTS) with a step resolution of $17 \mathrm{~nm}$ over a temperature-controlled rubber surface, which temperature can be externally controlled, as shown in the schematic diagram of Fig. 3. The sensor characterization has been performed by applying an axial strain of $5800 \mu \varepsilon$ in steps of $200 \mu \varepsilon$ each, and repeated at different temperatures from $36^{\circ} \mathrm{C}$ to $90^{\circ} \mathrm{C}$.

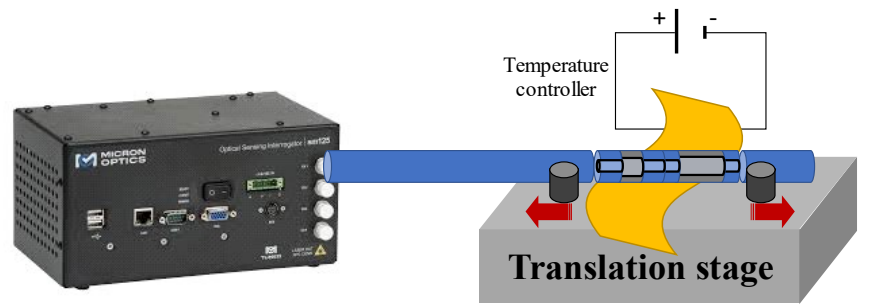

Fig. 3. Schematic diagram of the experimental setup.

\section{RESULTS AND DISCUSSIONS}

As in previous works [19], the principle of operation for this multiparameter sensor scheme was based on the combination of the received spectra by means of the FBG sensor interrogator in a reflection-working-mode operation and its fast Fourier transform (FFT) analysis. Fig. 4 illustrates the combined reflection spectrum of the proposed multiparameter sensor at room temperature when no strain was applied, measured by means of a commercial FBG sensors interrogator. 


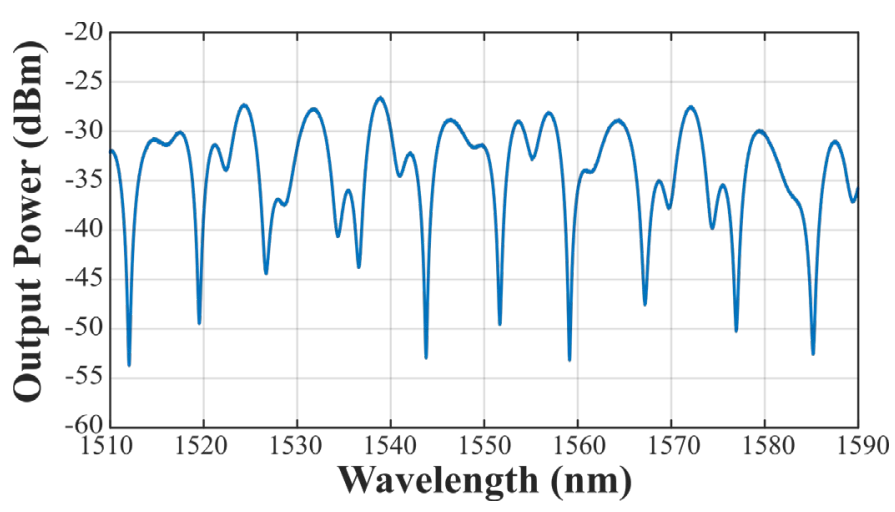

Fig. 4. Combined reflection spectrum of the multiparameter sensor at room temperature.

In order to discriminate each sensor information, the FFT of their spectra were analyzed as illustrated in Fig. 5, obtaining different contributions for each of the interferometers presented in this cascade FP-sensor. Tracking the phase of the FFT of those different spatial frequencies, the sensor changes can be easily monitored. These phase variations over these main peaks, (spatial frequencies of 0.1375 and $0.2875 \mathrm{~nm}^{-1}$ ), reflect the wavelength shifts induced by the strain or temperature in each spectral contribution separately.

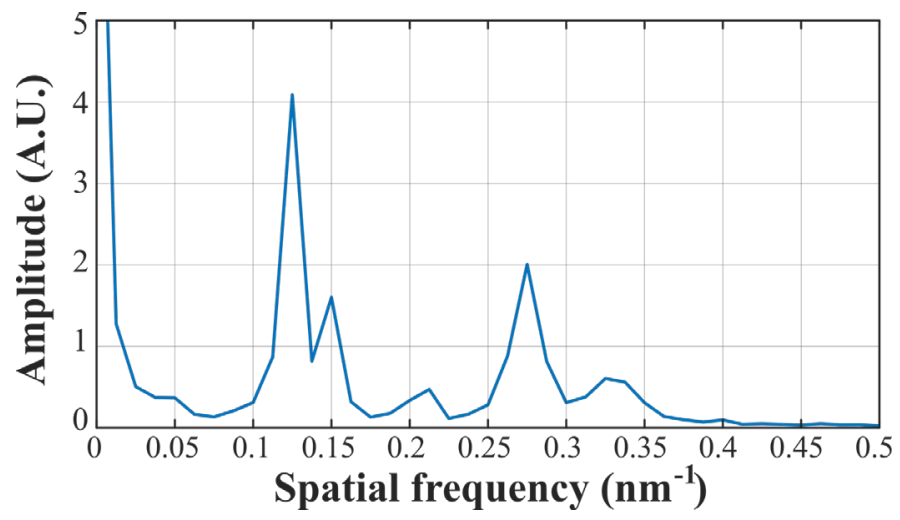

Fig. 5. Amplitude of the FFT from the spectrum of Fig. 4

The FBG interrogator records the combined interferometric signal reflected by the sensors at a refresh rate of $1 \mathrm{~Hz}$. In order to perform the measurement of the sensors in the FFT domain in real-time, a custom-made control software was employed [19]. The software logs the reflected spectra of the sensors, performs the FFT, and moves the MTS. As it has been previously pointed out, this process was repeated at different temperatures from $36^{\circ} \mathrm{C}$ to $90^{\circ} \mathrm{C}$.

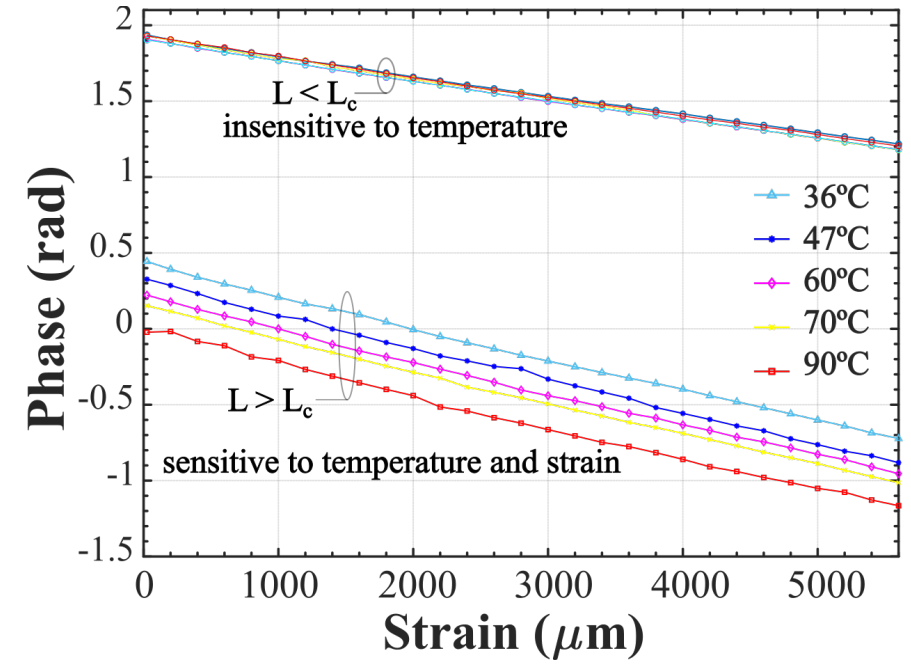

Fig. 6. Phase variation of the selected spatial frequencies as a result of strain and temperature variations.

Fig. 6 shows the phase variation of the selected spatial frequencies as a function of strain and temperature variations. In the upper part of this figure can be seen the behavior of the phase of the FFT for the selected spatial frequency related with the FPI, in other words, the cavity created by the interferometer with a HCF length shorter than the critical length. This interference is known to be insensitive to temperature but not to strain variations. That is the reason why it can be used for measuring axial strain. Here, the multiparameter sensor presents a clear linear response to strain variations with sensitivity of $-0.129 \mathrm{rad} / \mathrm{m} \varepsilon$ and an error of $\mathrm{R}^{2}=0.999$. On the other hand, this phase presents a negligible variation when temperature increases, as expected.

Likewise, when the HCF length exceeds the critical length, both FP interference and AR reflecting guidance appear in the reflection spectrum, being sensitive to parameters such as strain and temperature among others. That can be easily observed in the lower part of Fig. 6, where phase variations exist both for temperature and for strain modifications. Thus, only by tracking those values, it is possible to discriminate these two parameters independently.

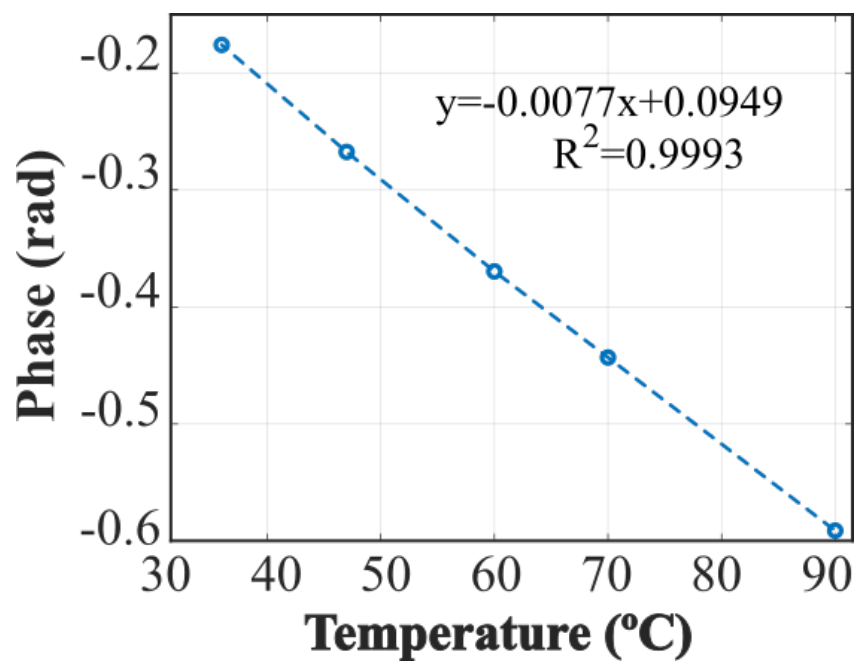


Fig. 7. Phase variations as a function of temperature.

In this case, the multiparameter sensor offers a remarkable linear response to strain variation with sensitivity of -0.2084 $\mathrm{rad} / \mathrm{m} \varepsilon$ and an error of $\mathrm{R}^{2}=0.999$, which means an improvement when this sensitivity value is compared with those obtained in previous studies [10]. In this case, as Fig. 7 shows, the response to temperature variation shows a sensitivity of $-0.0077 \mathrm{rad} /{ }^{\circ} \mathrm{C}$ and an error of $\mathrm{R}^{2}=0.999$.

\section{CONCLUSIONS}

In this work, a multiparameter sensor based on a multiinterferometric structure for temperature and strain measurements is presented and experimentally demonstrated. This structure, that is based on FP interference and AR reflecting guidance, was fabricated with two different air microcavities. The first one had a HCF length larger than the critical length, and the second one lower than this critical value that is $345 \mu \mathrm{m}$ and $165 \mu \mathrm{m}$ respectively. By tracking the phase of the FFT of the specific spatial frequencies, the sensor changes could be independently monitored. These phase variations follow the wavelength shifts induced by the strain or temperature at each spectral contribution separately. The attained experimental results show a strain and temperature sensitivities of $-0.2084 \mathrm{rad} / \mathrm{m} \varepsilon$ and $-0.0077 \mathrm{rad} /{ }^{\circ} \mathrm{C}$ respectively for the antiresonance contribution (the longer air micro-cavity), both with an error of $\mathrm{R}^{2}=0.999$. On the other hand, when analyzing the FP interference, a strain sensitivity of -0.129 $\mathrm{rad} / \mathrm{m} \varepsilon$ and an error of $\mathrm{R}^{2}=0.999$ was measured. This air microcavity was also demonstrated to be insensitive to temperature variations.

\section{ACKNOWLEDGMENT}

This work was supported by the Spanish AEI projects TEC2016-76021-C2 and PID2019-107270RB-C22, FEDER Funds, the European Union's Horizon 2020 research and innovation programme under the Marie Skłodowska-Curie grant agreement No 838143, Projects for young researches UPNA 2019, "Caja Navarra" and "la Caixa" foundations under agreement LCF/PR/PR13/51080004 and the grant BGP18/00180 of the "Beatriz Galindo" programme from the Spanish Ministry of Science, Innovation and Universities.

\section{REFERENCES}

[1] K. Markowski, K. Wojakowski, E. Pokropek, and M. Marzęcki, "Numerical and Experimental Performance Analysis of the Chirped Fiber Bragg Grating Based Abrasion Sensor for the Maintenance Applications in the Industry 4.0," Sensors, vol. 20, no. 3, pp. 770, Jan. 2020, $10.3390 / \mathrm{s} 20030770$

[2] A. Cachada, P.M. Moreira, L. Romero, J. Barbosa, P. Leitno, C.A.S. Gcraldcs, L. Deusdado, J. Costa, C. Teixeira, J. Teixeira, et al. "Maintenance 4.0: Intelligent and Predictive Maintenance System Architecture," in Proceedings of the 2018 IEEE 23rd International Conference on Emerging Technologies and Factory Automation (ETFA), Turin, Italy, 4-7 Sept. 2018; pp. 139-146.
[3] A. Schütze, N. Helwig, and T. Schneider, "Sensors 4.0 - smart sensors and measurement technology enable Industry 4.0," Journal of Sensors and Sensor Systems, vol. 7, no. 1, pp. 359-371, 2018, 10.5194/jsss-7-359-2018.

[4] C. Zhu, R. E. Gerald and J. Huang, "A Dual-Parameter Internally Calibrated Fabry-Perot Microcavity Sensor," IEEE Sensors Journal, vol. 20, no. 5, pp. 2511-2517, Mar. 2020, 10.1109/JSEN.2019.2954042.

[5] Z. Zhang, J. He, B. Du, F. Zhang, K. Guo, and Y. Wang, "Measurement of high pressure and high temperature using a dual-cavity Fabry-Perot interferometer created in cascade hollow-core fibers," Optics Letters, vol. 43, no. 24, pp. 6009-6012, 2018, 10.1364/OL.43.006009.

[6] Y. Zhang, J. Huang, X. Lan, L. Yuan, and H. Xiao "Simultaneous measurement of temperature and pressure with cascaded extrinsic FabryPerot interferometer and intrinsic Fabry-Perot interferometer sensors," Optical Engineering, vol. 53, no. 6, pp. 067101, 2014, 10.1117/1.OE.53.6.067101.

[7] C. Zhu, R. E. Gerald and J. Huang, "A Dual-Parameter Internally Calibrated Fabry-Perot Microcavity Sensor," IEEE Sensors Journal, vol. 20, no. 5, pp. 2511-2517, 2020, 10.1109/JSEN.2019.2954042.

[8] H. Gao, Y. Jiang, L. Zhang, Y. Cui, Y. Jiang, J. Jia, and L. Jiang, "Antiresonant mechanism based self-temperature-calibrated fiber optic Fabry-Perot gas pressure sensors," Optics Express, vol. 27, no. 16, pp. 22181-22189, 2019, 10.1364/OE.27.022181.

[9] P. Zhang, M. Tang, F. Gao, B. Zhu, S. Fu, J. Ouyang, P. P. Shum, and D. Liu, "Cascaded fiber-optic Fabry-Perot interferometers with Vernier effect for highly sensitive measurement of axial strain and magnetic field," Optics Express, vol. 22, no. 16, pp. 19581-19588, 2014, 10.1364/OE.22.019581.

[10]D. Leandro, M. Bravo, A. Ortigosa, and M. Lopez-Amo, "Real-time FFT Analysis for interferometric sensors multiplexing," Journal of Lightwave Technology, vol. 33, no. 2, pp. 354-360, 2015, 10.1109/JLT.2014.2388134.

[11]M. Galarza, R. A. Perez-Herrera, D. Leandro, A. Judez, M. Lopez-Amo, "Spatial-frequency multiplexing of high-sensitivity liquid level sensors based on multimode interference micro-fibers," Sensors and Actuators A: Physical, vol. 307, 111985, 2020, 10.1016/j.sna.2020.111985.

[12]D. Leandro, M. Bravo, A. Ortigosa et al., "Monitoring multiple interferometric sensors multiplexed in a single fiber loop mirror, " in Proc. SPIE 9634, 24th International Conference on Optical Fibre Sensors, 963418, 2015.

[13]D. Leandro, M. Bravo, and M. Lopez-Amo, "High resolution polarization independent high-birefringence fiber loop mirror sensor," Optics Express, vol. 23, no. 24, pp. 30985-30990, 2015, 10.1364/OE.23.030985.

[14]C. Han, H. Ding, H. and F. Lv "Demonstration of a refractometric sensor based on an optical micro-fiber three-beam interferometer," Scientific Reports, vol. 4, pp. 7504, Dec. 2014, 10.1038/srep07504.

[15]R.A. Pérez Herrera, S. Novais, M. Bravo, D. Leandro, S. Silva, O. Frazao, and M. Lopez-Amo "," in Proc. SPIE 11199, Seventh European Workshop on Optical Fibre Sensors EWOFS, Limassol, Cyprus, 2019, pp. 1119925 , $10.1117 / 12.2540150$

[16]P.R. Horche, M. López Amo, M.A. Muriel, J.A. Martín Pereda, "Spectral behavior of a low cost all fiber component based on untapered multifiber unions," IEEE Photonics Technology Lett., vol. 1, no. 7, pp. 184-187, Jul. $1989,10.1109 / 68.36033$

[17]X. Zhang, H. Pan, H. Bai, M. Yan, J. Wang, C. Deng, and T. Wang, "Transition of Fabry-Perot and antiresonant mechanisms via a SMFcapillary-SMF structure," Optics Letters, vol. 43, no. 10, pp. 2268-2271, 2018, 10.1364/OL.43.002268.

[18]F. Hou, X. Zhang, H. Pan, W. Sun, L. Yang, C. Deng, and T. Wang "Investigation of transmission and reflection of antiresonance in a SMFcapillary-SMF structure," in Proc. SPIE 10821, Advanced Sensor Systems and Applications VIII, 108211O, 2018, 10.1117/12.2501175.

[19]D. Leandro, M. Bravo, A. Ortigosa, M. Lopez-Amo, "Real-time FFT analysis for interferometric sensors multiplexing," Journal of Lightwave Technology, vol. 33, no. 2, pp. 354-360, Jan. 2015, 10.1109/JLT.2014.2388134 


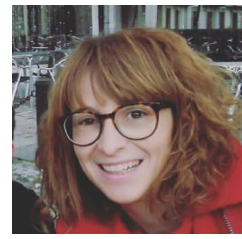

Rosa Ana Pérez-Herrera was born in Cantabria, Spain, in February 1979. She received the telecommunications engineering degree from the University of Cantabria, Spain, in 2004. In 2005 she joined the Optical Communications Group at the Department of Electrical and Electronic Engineering of the Public University of Navarra (Pamplona, Spain). During this period, she was a visiting Ph.D. student at INESC in Unidade De Optoelectronica E Sistemas Electronicos in Porto (Portugal), at the School of Engineering and Mathematical Sciences at City University of London and at the Department of Information Engineering at Parma University (Italy) among others. In 2009, she became an Assistant Professor in the Electrical and Electronic Engineering department of the Public University of Navarra. In 2010 she obtained her Ph.D. degree from the Public University of Navarra, Spain. Her research interests are in Raman amplifiers, erbium-doped amplifiers, fiber-optic sensors and multiplexing architectures.

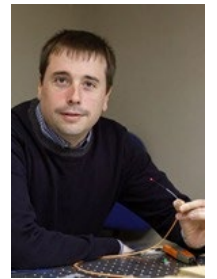

Mikel Bravo Acha received the Ph.D degree from the Universidad Pública de Navarra in February 2015. The topic of his doctoral dissertation was on photonics systems for optical fiber sensors and fiber based optical sources. He was research visitor in the INESC Porto and the City University London laboratories. He also did a post-doctoral period in Quebec exploring the integrated optics field. Nowadays, he is collaborating with the Optical Communations Group of the Universidad Pública de Navarra. He is author or co-author of over 40 papers in international refereed journals and conference contributions. His current research interests include interferometric optical fiber sensors, the random distributed feedback effect for optical sources, optical sensing, integrated optics and its application in real environments.

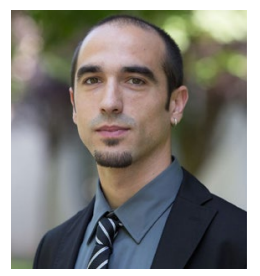

Daniel Leandro received the M.Sc. in telecommunication engineering, the M.Eng. in communications and the Ph.D. degree from the Universidad Publica de Navarra, Pamplona, Spain, in 2010, 2012, and 2016, respectively. He has been a visiting researcher among others in the School of Frontier Sciences, The University of Tokyo, Tokyo, Japan, in 2018. His research interests include fiber optic lasers, fiber optic sensors, sensor networks, and multiplexing architectures. $\mathrm{He}$ is currently a researcher in the Institute of Smart Cities (ISC) in the Universidad Pública de Navarra.

Susana Novais received the M.Sc. degree in biomedical engineering in 2012, from the Bragança Polytechnic Institute, Bragança, Portugal. She is currently working toward the Ph.D. degree in the Physics Department, University of Aveiro, Aveiro, Portugal. Her main research interests are optical fiber sensors applied to the lithium ion batteries and optical fiber designs, and their applications in chemically challenging media.

Javier Pradas received the telecommunication engineering degree from the Universidad Publica de Navarra, Spain in 2020.

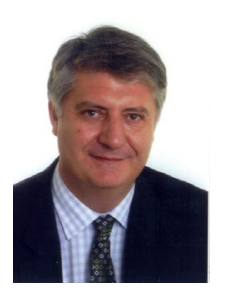

Manuel Lopez-Amo (M'91, SM '98) is a fullprofessor in photonics at Universidad Pública de Navarra from 1996. He is the head of the Optical Communications group of this University. His research Interests are in optical fiber sensors, optical networks, optical fiber lasers and integrated optics. MLA is the author of more than $170 \mathrm{JCR}$ peer reviewed technical articles, 4 patents, 8 book chapters and more than 140 communications at international conferences MLA has served in 32 International and 15 national technical committees of well reputed conferences, being now a member of the technical committees of the International Conference on fiber optic sensors (OFS) and the European Workshop on optical fiber sensors (EWOFS), among others. MLA has been Chairman of the Optoelectronic Committee of Spain. MLA has been the director of the Engineering Faculty and of the Electrical and Electronic department of Universidad Pública de Navarra. Professor Lopez-Amo has led 33 research projects (International, national and with companies) being coinvestigator in another 26. He is a Senior member of IEEE and member of the OSA. 